\title{
NEOPLASIA PAPILAR INTRAQUÍSTICA DE MAMA DE ASPECTO BENIGNO CONTENIENDO UN CARCINOMA DUCTAL IN SITU
}

\begin{abstract}
Ivan Eduardo Pinto Larrea 1,a, Magaly Malca Tocas 1,b, Gilmar Grisson Barron ${ }^{1, b}$, Miguel Matute Correa $^{1, b}$, Manuel Fernandez Lopez ${ }^{1, c}$, Roger V. Araujo Castillo ${ }^{2, d}$

RESUMEN

El cáncer de mama es una patología prevalente; la neoplasia papilar intraquistica representa solo el $1 \%$ de todos ellos, pueden ser divididos en infiltrantes y no infiltrantes. Se presenta el caso de una mujer de 46 años, que acude por un tumor de mama izquierda sospechoso, con biopsia core sin evidencia de carcinoma, se decidió realizar una Resección local radical de tumor con diagnóstico papiloma intraductal con carcinoma ductal in situ, se realizó cirugía de ampliación de márgenes y biopsia de ganglio centinela con técnica de azul patente con resultado negativo, seguida de Radioterapia. Este reporte resalta la importancia de considerar que las lesiones papilares pueden albergar lesiones malignas, por lo que es recomendable realizar exéresis completa de la lesión para realizar un tratamiento adecuado.
\end{abstract}

Palabras claves: Neoplasia papilar intraquística; Carcinoma ductal in situ(Fuente: DeCS BIREME)

\section{INTRACYSTIC PAPILLARY BREAST NEOPLASIA WITH BENIGN APPEARANCE CONTAINING IN SITU DUCTAL CARCINOMA.}

\begin{abstract}
Breast cancer is a prevalent pathology; the intracystic papillary neoplasm represents only $1 \%$ of all of them, they can be divided into infiltrants and in situ. We present the case of a 46-year-old woman, who camed with a suspicious left breast nodule, with a core biopsy without evidence of carcinoma, and decided to perform a radical local resection of the tumor with an intraductal papilloma diagnosis with ductal carcinoma in situ. The patient underwent re-resection surgery and sentinel lymph node biopsy with blue patent technique with negative result, followed by Radiotherapy. This report highlights the importance of considering that papillary lesions may contain malignant lesions, so it is recommended to perform complete resection of the lesion to carry out an adequate treatment.
\end{abstract}

Keywords: Intracystic Papillary Neoplasm; Ductal carcinoma in situ(Source: MeSH NLM)

\section{INTRODUCCIÓN}

El cáncer de mama es el cáncer más frecuente en las mujeres tanto en países desarrollados como en países en desarrollo. La incidencia en el año 2018 fue de 56.8 por 100000 mujeres en países sudamericanos y la mortalidad fue de 13.4 × 100000 mujeres en países sudamericanos $^{1}$. Según el Registro de Cáncer de Lima Metropolitana 2010-2012 se presentaron 6030 casos nuevos con una incidencia de 40.9 casos por 100000 mujeres, representando el $18.3 \%$ de todas las neoplasias malignas en mujeres, con una tasa de Mortalidad de 10.7 por 100000 mujeres $^{2}$.
La neoplasia papilar intraquistica es un cáncer de mama poco común, que representa solo el 0.5 al $1 \%$ de todos los cánceres de mama ${ }^{3-6}$. Los carcinomas papilares pueden ser divididos en infiltrantes y no infiltrantes. Los carcinomas papilares son caracterizados por un crecimiento papilar dentro de un quiste y puede ser asociado a un carcinoma ductal in situ $(46 \%)$ o con un carcinoma infiltrante $(36 \%)^{7}$. La ausencia de células mioepiteliales en la periferia de las áreas del tumor papilar han sido utilizadas histológicamente para determinar la naturaleza infiltrante de la lesión.

Generalmente ocurre en mujeres postmenopausicas, con

\footnotetext{
Servicio de Oncología Ginecológica, Departamento de Gíneco-Obstetricia, Hospital Nacional Edgardo Rebagliati Martins, EsSalud, Lima-Perú

Dirección de Investigación, Instituto de Evaluación de Tecnologías en Salud e Investigación (IETSI), EsSalud, Lima-Perú

Médico Especialista en Gineco-Obstetricia, Fellow en Ginecología Oncológica

Médico Especialista en Ginecología Oncológica

Jefe del Servicio de Ginecologia Oncologica

Médico Epidemiólogo

Citar como: Pinto-Larrea IE, Malca-Tocas M, Grisson G, Matute M, Fernandez M, Araujo-Castillo RV. Neoplasia papilar intraquística de mama de aspecto benigno conteniendo un carcinoma ductal in situ. Rev Peru Investig Matern Perinat. 2019;8(4): 54-8

DOI https://doi.org/10.33421/inmp.2019174
}

Recibido: 10-10-2019 Aceptado: 12-12-2019 
presentación subclínica de aparición de tumor no doloroso o con descarga a través del pezón. Radiológicamente se evidencia una lesión circunscrita y a la ecografía un quiste complejo con componente sólido con vascularidad en el interior del componente sólido ${ }^{4,8}$. El diagnóstico citológico puede generar falsos negativos, la biopsia trucut ecoguiada de la parte sólida puede dar un diagnóstico erróneo que debe corroborarse con la exéresis de la lesión ${ }^{9}$. Puede tener diferentes manejos de acuerdo a los hallazgos patológicos: cirugía, cirugía con radioterapia o cirugía, radioterapia y tratamiento sistémico. Este tipo de tumor tiene baja tasa de recurrencia $(7.8 \%)^{8}$. La sobrevida específica es alrededor de $100 \%$ y la sobrevida libre de enfermedad es $96 \%, 85 \%$ y $77 \%$ a los 2,5 y 10 años respectivamente ${ }^{10}$

El reconocimiento de los carcinomas papilares como una entidad maligna por parte de los patólogos es alto, pero la concordancia de su clasificación en enfermedad in situ e invasiva puede ser problemática ${ }^{6,8,11-13}$, al igual que la evaluación de factores pronósticos y predictivos en el componente invasivo. Por estas razones, se reporta un caso de carcinoma papilar intraquistico ocurrido en el Hospital Edgardo Rebagliati Martins en el año 2017.
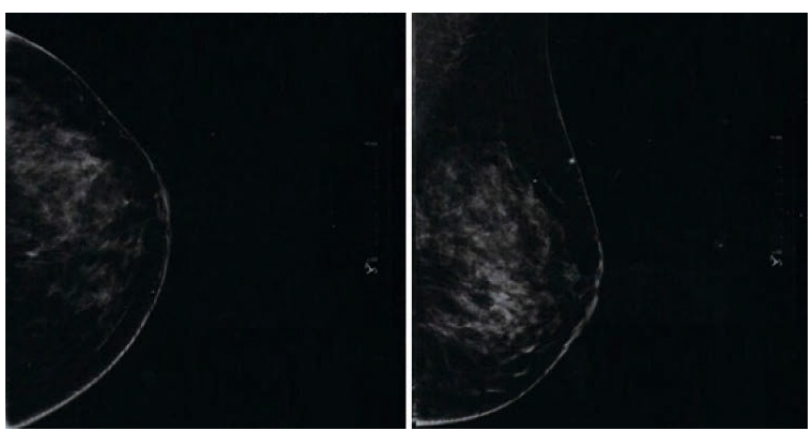

Figura 1. Mamografía: Mama izquierda con distorsión de la arquitectura en tercio posterior de radio 2 , localizado a $6 \mathrm{~cm}$ del pezón (Birads 4B)
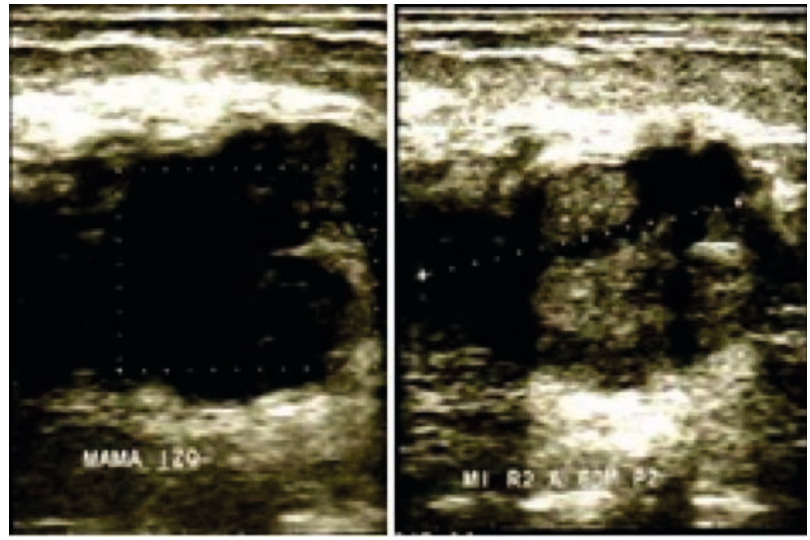

Figura 2. Ecografía de mamas: Mama izquierda con extensa lesión intraquistica multilobulada con vascularidad interna de $3 \mathrm{~cm}$ (Birads 4B)

\section{REPORTE DE CASO}

Paciente de 46 años con antecedentes de diabetes 2 e hipotiroidismo bajo control, sin antecedentes familiares de cáncer, presenta un tumor de mama izquierda desde hace 2 años, con crecimiento progresivo del tumor, sin presentar dolor ni otro síntoma asociado. Al examen físico, se evidencio una tumoración de $4 \times 4 \mathrm{~cm}$ en el cuadrante superior externo, sin afectación de ganglios axilares. La mamografía mostró una distorsión de la arquitectura en tercio posterior de radio 2 , localizado a $6 \mathrm{~cm}$ del pezón y fue catalogado como Birads 4B (Figura 1). La ecografía de mamas evidenció una extensa lesión intraquistica multilobulada con vascularidad interna de $3 \mathrm{~cm}$ - Birads 4B (Figura 2). Se realizó Biopsia Core con resultado histopatológico de Neoplasia Papilar con proliferación Epitelial, con inmunohistoquímica (resultados) que favorece diagnóstico de papiloma intraductal intraquístico con focos de hiperplasia ductal. No hubo evidencia de carcinoma ductal. Se decide realizar una Resección local radical de tumor.
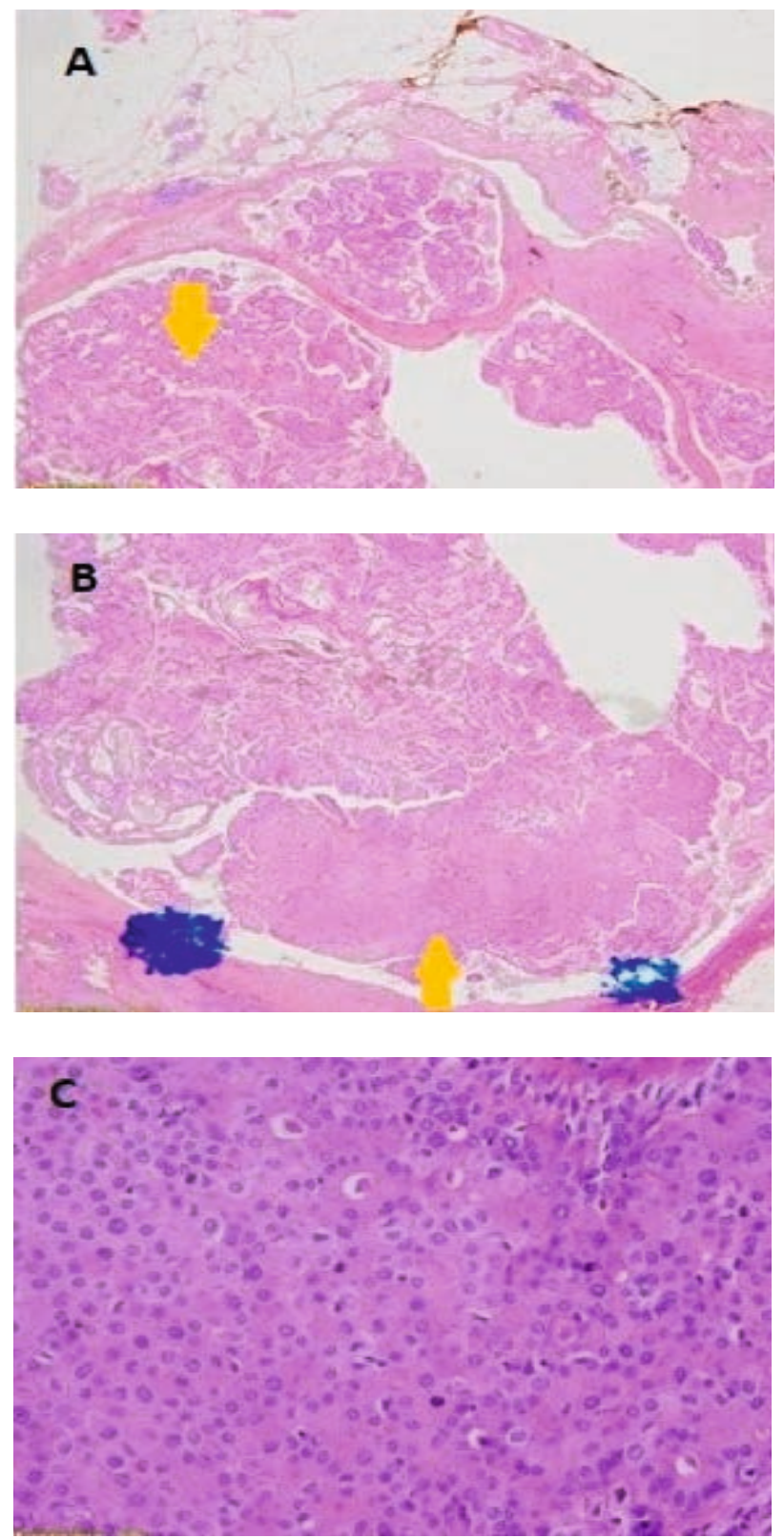

Figura 3. Patología: A) tumoración de aspecto quístico con proyecciones papilares hacia el interior (flecha). B) Papiloma intraductal con foco de carcinoma ductal in situ (flecha). C) Carcinoma ductal con grado nuclear intermedio, patrón sólido y cribiforme 

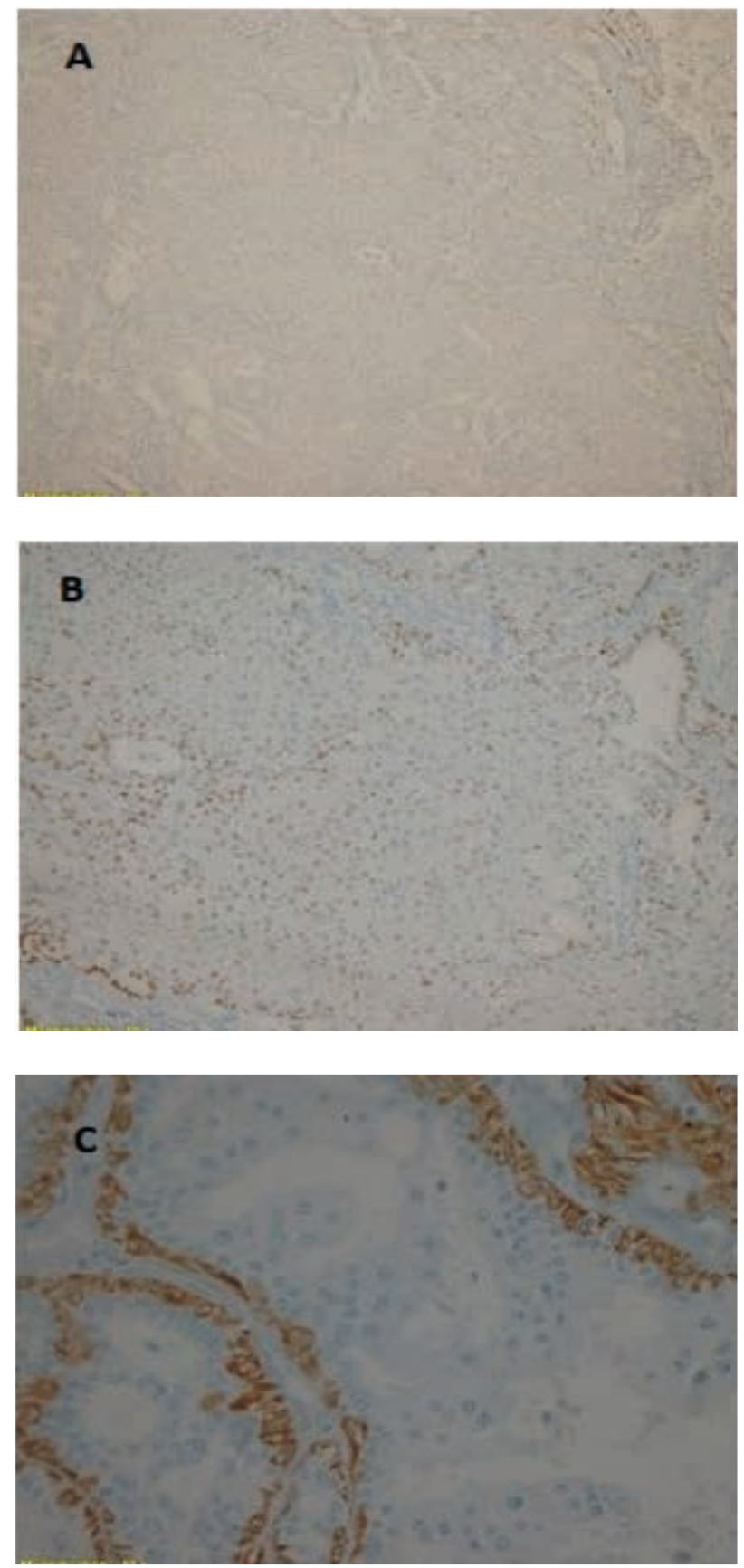

Figura 4. Inmunohistoquímica: A) Receptores de estrógeno negativo, B) Receptores de progesterona positivo y C) Calponina positivo

\section{Características Patológicas de la pieza quirúrgica}

Se obtuvo una tumoración de $23 \times 17 \times 10 \mathrm{~mm}$, de aspecto quístico con proyecciones papilares parduscas hacia el interior, en contacto aparente con los bordes anterior, posterior y externo (Figura 3A). Histológicamente se apreció papiloma intraductal, pero acompañado de carcinoma ductal in situ de $5 \mathrm{~mm}$ de dimensión, grado nuclear intermedio, patrón sólido y cribiforme (Figura 3B y $3 C$ ). El estudio de Inmunohistoquímica mostró Receptor de estrógeno negativo, Receptor de Progesterona (1+), y Calponina positivo en células mioepiteliales descartando la posibilidad de microinfiltración (Figura 4).
Dado que la lesión estuvo a $1 \mathrm{~mm}$ del borde anterior, la junta médica decidió que la paciente tuviera una nueva cirugía de ampliación de márgenes y biopsia de ganglio centinela con técnica de azul patente. La nueva cirugía revelo proliferación epitelial con hiperplasia ductal usual y no afectación de ganglios axilares, por lo que el estadiaje clínico fue 0 . Basado en la edad, el diagnóstico histológico, y el hecho que se conservó la mama, la paciente recibió Radioterapia adyuvante (50Gy/25 fracciones) + Boost al lecho quirúrgico (16Gy/8 fracciones). La paciente no recibió hormoterapia ni quimioterapia. Al momento de este reporte la paciente tiene un mes de finalizada radioterapia, continua en controles por el servicio, y no tienen evidencia de recurrencia.

\section{DISCUSIÓN}

Este caso ilustra como una lesión quística compleja con biopsia inicial negativa para neoplasia maligna puede albergar lesiones malignas, especialmente en casos de lesiones papilares.

El carcinoma papilar intraquístico es generalmente una neoplasia maligna de mujeres de la tercera, edad, sin embargo, en este caso afecto a una mujer premenopausica, corroborando los hallazgos descritos por Grabowski et al ${ }^{14}$ que en una revisión de 917 casos, encontró afectación de mujeres de todas las edades. Clínicamente se presenta generalmente como una tumoración indolora, ovalada y pequeña con historia de descarga de secreción sanguinolenta a través del pezón o como lesiones más grandes de aspecto ovalado ${ }^{4}$, en nuestro caso se presentó como una tumoración de crecimiento progresivo indolora, lejana del complejo areola pezón y sin descarga a través del pezón por ser una lesión papilar periférica, las cuales son lesiones más raras, localizadas en la periferia del sistema ductal dentro de la unidad ductolobular, las cuales tienen mayor riesgo de atipia celular y de coexistencia de lesiones malignas entre $10-30 \%{ }^{15}$.

Respecto a los hallazgos de imágenes en nuestro caso se evidenció en la mamografía una distorsión de arquitectura en tercio posterior, no bien circunscrita, localizada en el cuadrante supero externo y en la ecografía se evidenció una lesión intraquística multilobulada con vascularización interna sospechosa de lesión maligna. Según la literatura ${ }^{4,15}$ generalmente se evidencia una lesión única circunscrita ovalada o lobulada en la mamografía en aquellas lesiones grandes y en el ultrasonido se evidencia hasta 3 tipos de patrones: una masa intraductal con o sin dilatación del ducto, una masa intraquística y una tumoración sólido quística con predominio del patrón sólido dentro de un ducto; cuyo componente sólido con alto flujo vascular, que indican sospecha de lesión maligna, similar a lo encontrado en nuestro caso.

En esta ocasión, se realizó una biopsia trucut con resultado final papiloma intraductal intraquístico con foco de hiperplasia. La literatura menciona que se puede realizar biopsia del componente sólido, con aguja fina o biopsia trucut, ambas con guía ecográfica; sin embargo, se suele obtener alrededor de $20 \%$ de falsos negativos. Aun así, se debe tener en cuenta que cuando el componente sólido es biopsiado en su parte central, la lesión neoplásica aún puede encontrarse en las regiones periféricas, por lo que se recomienda la biopsia escisional de toda la lesión $4,15,16$. En nuestro caso, a pesar de realizarse la biopsia con guía 
ecográfica y tomarse múltiples muestras, la patología no mostro evidencia de cáncer.

Se realizó la escisión completa de la lesión obteniéndose como resultado un papiloma intraductal con un foco de carcinoma in situ, al que se le realizó pruebas de inmunohistoquímica descartando micro-infiltración. Según la literatura ${ }^{9}$ una vez diagnosticada una lesión papilar, es difícil evidenciar si la lesión es benigna, atípica o maligna; y las lesiones malignas son difíciles de clasificar en una lesión in situ, una infiltrante o la combinación de ambas. Justamente la inmunohistoquimica puede ayudar a diferenciar micro-infiltración $n^{4,7,13,17}$ como ocurrió en nuestro caso. Los carcinomas papilares intraquísticos usualmente son de grado nuclear bajo o intermedio, sin evidencia de necrosis, con receptores de estrógenos positivos y con c-erbB2 negativos ${ }^{4,7}$. En el presente caso fue un carcinoma de grado nuclear intermedio, sin evidencia de necrosis y con receptores de estrógeno negativos.

Existen diferentes formas de tratamiento según la literatura. La base del tratamiento es la escisión quirúrgica de la lesión; tanto la cirugía de conservación como la mastectomía (simple o radical modificada) han sido descritas en los reportes de casos para tratar el carcinoma papilar intraquístico, teniendo un buen pronóstico con todas las intervenciones ${ }^{4,7,14,18}$. Sin embargo, existen reportes de afectación axilar o metástasis cuando se asocian a un componente infiltrante ${ }^{19}$. Tras el tratamiento quirúrgico, varios estudios han reportado el uso de radioterapia adyuvante, asociada o no a terapia hormonal. Sin embargo, el rol de la radioterapia en estos pacientes aún no está definido ${ }^{10}$, pues no cambia la incidencia de recurrencia local ni la tasa de sobrevida global en los grupos que recibieron radioterapia comparado con el grupo que no recibió radioterapia. Respecto al uso de hormonoterapia, a pesar de contar con receptores hormonales positivos, se ha visto en los estudios que el uso de terapia hormonal no tendría un mejor resultado, por lo que algunos autores ${ }^{4,9,14}$ no recomiendan el uso de tratamiento adyuvante para mejorar la sobrevida libre de enfermedad, a menos que se encuentre asociado un componente infiltrante. Respecto al estudio de la axila en estos pacientes, se estima entre un $8-12 \%$ de casos que tuvieron afectación axilar ${ }^{14}$ por lo que se sugiere realizar biopsia de ganglio centinela a pesar de la baja tasa de afectación axilar.

En el presente caso, la paciente recibió cirugía de conservación con biopsia de ganglio centinela, logrando obtener bordes libres y descartándose la presencia de afectación axilar, asimismo se decidió dar tratamiento adyuvante con radioterapia al lecho quirúrgico con boost asociado.

Según la literatura esta forma de cáncer de mama es considerada de bajo riesgo y de buen pronóstico, con crecimiento lento y con tasas de sobrevida global de $100 \%$ a los 10 años y de sobrevida libre de enfermedad de hasta $91 \%{ }^{18}$.

En conclusión, es importante tener sumo cuidado con las lesiones papilares así tengan aspecto benigno, ya que pueden albergar hasta un $20 \%$ de lesiones malignas, tal como ilustra nuestro caso. Por lo tanto, es siempre recomendable realizar exéresis completa de la lesión, ya que el tratamiento final podría ser bastamente diferente.
Financiamiento: Este estudio no requirió financiamiento

Conflictos de interés: Los autores declaran no tener conflictos de interés respecto al contenido de esta investigación.

\section{REFERENCIAS BIBLIOGRÁFICAS}

1. Bray F, Ferlay J, Soerjomataram I, Siegel RL, Torre LA, Jemal A. Global cancer statistics 2018: GLOBOCAN estimates of incidence and mortality worldwide for 36 cancers in 185 countries. CA: A Cancer Journal for Clinicians [Internet]. noviembre de 2018 [citado 21 de noviembre de 2018];68(6):394-424. Disponible en: http://doi.wiley. com/10.3322/caac.21492

2. Payet, E, Pérez, P, Poquioma, E, Díaz, E. Registro de Cáncer de Lima Metropolitana - Incidencia y Mortalidad 2010-2012 [Internet]. Departamento de Epidemiologia y Estadistica del Cáncer - Instituto Nacional de Enfermedades Neoplásicas; 2016. Disponible en: http://www.inen.sld.pe/ portal/documentos/pdf/banners_2014/2016/Registro\%20 de $\% 20$ C $\%$ C $3 \%$ A 1 ncer $\% 20$ Lima $\% 20$ Metropolitana $\% 20$ 2010\%20-\%202012_02092016.pdf

3. Suh YJ, Shin H, Kwon TJ. Natural History of Invasive Papillary Breast Carcinoma Followed for 10 Years: A Case Report and Literature Review. Case Reports in Medicine [Internet]. 2017 [citado 21 de noviembre de 2018];2017:1-4. Disponible en: https://www.hindawi.com/journals/crim/2017/3725391/

4. Reefy SA, Kameshki R, Sada DA, Elewah AA, Awadhi AA, Awadhi KA. Intracystic papillary breast cancer: a clinical update. Ecancermedicalscience. 2013;7:286.

5. Romics L, O'Brien ME, Relihan N, O'Connell F, Redmond HP. Intracystic papillary carcinoma in a male as a rare presentation of breast cancer: a case report and literature review. Journal of Medical Case Reports [Internet]. diciembre de 2009 [citado 21 de noviembre de 2018];3(1). Disponible en: http://jmedicalcasereports.biomedcentral. com/articles/10.1186/1752-1947-3-13

6. Pant I, Joshi S. Invasive papillary carcinoma of the male breast: Report of a rare case and review of the literature. Journal of Cancer Research and Therapeutics [Internet]. 2009 [citado 21 de noviembre de 2018];5(3):216. Disponible en: http://www.cancerjournal.net/text.asp?2009/5/3/216/57132

7. Muñoz, M, Martin, S, Nieto, M, Noguero, R, Rodriguez, I. Carcinoma papilar intraquístico de mama con focos de infiltración. Presentación de dos casos. Colombia Médica [Internet]. 2012 [citado 20 de octubre de 2018];43:1626. Disponible en: http://www.redalyc.org/articulo. oa? id $=28323195009$

8. Pal SK, Lau SK, Kruper L, Nwoye U, Garberoglio C, Gupta RK, et al. Papillary carcinoma of the breast: an overview. Breast Cancer Research and Treatment [Internet]. agosto de 2010 [citado 21 de noviembre de 2018];122(3):637-45. Disponible en: http://link.springer.com/10.1007/s10549-0100961-5

9. Collins LC, Schnitt SJ. Papillary lesions of the breast: selected diagnostic and management issues: Papillary breast lesions. Histopathology [Internet]. 13 de diciembre de 2007 [citado 21 de noviembre de 2018];52(1):20-9. Disponible en: http:// doi.wiley.com/10.1111/j.1365-2559.2007.02898.x

10. Solorzano CC, Middleton LP, Hunt KK, Mirza N, Meric F, Kuerer HM, et al. Treatment and outcome of patients with intracystic papillary carcinoma of the breast. The American Journal of Surgery [Internet]. octubre de 2002 [citado 21 de noviembre de 2018];184(4):364-8. Disponible en: http:// linkinghub.elsevier.com/retrieve/pii/S0002961002009418 
11. Ibarra JA. Papillary Lesions of the Breast. The Breast Journal [Internet]. mayo de 2006 [citado 21 de noviembre de 2018];12(3):237-51. Disponible en: http://doi.wiley. com/10.1111/j.1075-122X.2006.00248.x

12. Masood S, Loya A, Khalbuss W. Is core needle biopsy superior to fine-needle aspiration biopsy in the diagnosis of papillary breast lesions? Diagnostic Cytopathology [Internet]. junio de 2003 [citado 21 de noviembre de 2018];28(6):32934. Disponible en: http://doi.wiley.com/10.1002/dc.10251

13. Rakha EA, Ellis IO. Diagnostic challenges in papillary lesions of the breast. Pathology [Internet]. enero de 2018 [citado 21 de noviembre de 2018];50(1):100-10. Disponible en: https:// linkinghub.elsevier.com/retrieve/pii/S0031302517305184

14. Grabowski J, Salzstein SL, Sadler GR, Blair S. Intracystic papillary carcinoma: A review of 917 cases. Cancer [Internet]. 1 de septiembre de 2008 [citado 5 de diciembre de 2018];113(5):916-20. Disponible en: http://doi.wiley. com/10.1002/cncr.23723

15. Ganesan S, Karthik G, Joshi M, Damodaran V. Ultrasound spectrum in intraductal papillary neoplasms of breast. The British Journal of Radiology [Internet]. octubre de 2006 [citado 5 de diciembre de 2018];79(946):843-9. Disponible en: http://www.birpublications.org/doi/10.1259/bjr/69395941

16. Levine $\mathrm{PH}$, Waisman J, Yang GCH. Aspiration cytology of cystic carcinoma of the breast. Diagnostic Cytopathology [Internet]. enero de 2003 [citado 5 de diciembre de 2018];28(1):39-44. Disponible en: http://doi.wiley. com/10.1002/dc.10209
17. Tse GM, Tan PH, Moriya T. The role of immunohistochemistry in the differential diagnosis of papillary lesions of the breast. Journal of Clinical Pathology [Internet]. 1 de mayo de 2009 [citado 21 de noviembre de 2018];62(5):407-13. Disponible en: http://jcp.bmj.com/cgi/doi/10.1136/jcp.2008.063016

18. Lefkowitz M, Lefkowitz W, Wargotz ES. Intraductal (intracystic) papillary carcinoma of the breast and its variants: A clinicopathological study of 77 cases. Human Pathology [Internet]. agosto de 1994 [citado 27 de diciembre de 2018];25(8):802-9. Disponible en: http://linkinghub. elsevier.com/retrieve/pii/004681779490250X

19. Okita R, Ohsumi S, Takashima S, Saeki T, Aogi K, Nishimura $R$. Synchronous liver metastases of intracystic papillary carcinoma with invasion of the breast. Breast Cancer [Internet]. octubre de 2005 [citado 27 de diciembre de 2018];12(4):327-30. Disponible en: http://link.springer. com/10.2325/jbcs. 12.327

\section{Correspondencia:}

Ivan Eduardo Pinto Larrea

Dirección: Av. Edgardo Rebagliati N 490, Jesús María. Lima, Perú, Teléfono: (01) 2654901 - anexo 3141

Correo electrónico: ivanpinto8602@gmail.com 